\title{
Self-seeded four-wave mixing cascades utilizing fiber Bragg grating
}

\begin{abstract}
Utilizing fiber Bragg grating (FBG), we demonstrate self-seeded four-wave mixing (FWM) cascades. Larger FWM cascades are produced at higher erbium-doped fiber amplifier (EDFA) output power using a simple scheme. Six channels are generated at the EDFA output power of $252.35 \mathrm{~mW}$. The separation gap of selective dual wavelength is designed at $1.6 \mathrm{~nm}$, following to the wavelength separation of the FBGs used.
\end{abstract}

Keyword: Fiber lasers; Four-wave mixing cascades; Optics nonlinearities 